The University of Southern Mississippi

The Aquila Digital Community

Faculty Publications

$2-1-2017$

\title{
I Love My Baffling, Backward, Counterintuitive, Overly Complicated Magnitudes
}

Christopher Sirola

University of Southern Mississippi, Christopher.Sirola@usm.edu

Follow this and additional works at: https://aquila.usm.edu/fac_pubs

Part of the Physics Commons

\section{Recommended Citation}

Sirola, C. (2017). I Love My Baffling, Backward, Counterintuitive, Overly Complicated Magnitudes. The Physics Teacher, 55, 15-16.

Available at: https://aquila.usm.edu/fac_pubs/15856

This Article is brought to you for free and open access by The Aquila Digital Community. It has been accepted for inclusion in Faculty Publications by an authorized administrator of The Aquila Digital Community. For more information, please contact Joshua.Cromwell@usm.edu. 


\section{I love my baffling, backward, counterintuitive, overly com- plicated magnitudes}

Christopher Sirola, Associate Professor, Department of Physics \& Astronomy, University of Southern Mississippi; Christopher.Sirola@ usm.edu

A ll professions have their jargon. But astronomy goes the extra parsec. Here's an example. Vega, one of the brighter stars in the night sky, has an apparent magnitude (i.e., an apparent brightness) of approximately zero. Polaris, the North Star, has an apparent magnitude of about +2 . Despite this, Vega appears brighter than Polaris, and not by two, but by a factor of about six times.

How do we teach these odd quantities to students? Never mind that-for a non-astronomer, how does one teach these to oneself?

Astronomy probably isn't the oldest profession (ahem), but it may be the oldest science, if we think of science involving detailed theorizing and copious data taking. The nice thing about this is that astronomers have a long history to draw upon. The problem is also that astronomers have a long history to draw upon.

The first person we know of who attempted a systematic reckoning of star brightnesses was a Greek named Hipparchus, who lived in the second century B.C.E. He devised a scheme where the 20 brightest stars to the naked eye (and in his time naked eye observations were the only option) were of the first magnitude. The next set of brightness for stars was of the second magnitude, and so on down to those of the sixth magnitude, which consisted of stars barely visible at all.

It's not known for sure why he chose this way to express star brightnesses, though the modern term magnitude may give us a clue. Magnitude shares the same root as magnificent and magnate, all of these deriving from the Latin magnitudo, or "greatness." So it is often thought that Hipparchus linked importance to brightness in his scheme.

Fast-forward to the 1850s, where an English astronomer named Norman Pogson put Hipparchus' magnitudes on a strict mathematical basis. Pogson kept the notion that smaller positive numbers-and by extension, negative numbersmeant brighter stars, and set a difference of five magnitudes equivalent to exactly 100 times in brightness. In this system, a star cited as having a -2 apparent magnitude is 100 times brighter than a star with a +3 apparent magnitude. Astronomers of course interpolate to find brightness factors for other differences in magnitudes (see Table I). Note what we've done-magnitudes are dealt with by subtraction whereas brightnesses are dealt with by division.
Table I. How a sample of magnitude differences translate into factors of brightnesses.

\begin{tabular}{|c|c|}
\hline \multicolumn{2}{|c|}{ Comparing Magnitudes to Brightnesses } \\
\hline $\begin{array}{c}\text { Difference in } \\
\text { magnitudes }\end{array}$ & $\begin{array}{c}\text { Factor of } \\
\text { brightness }\end{array}$ \\
\hline 1 & 2.5 \\
\hline 2 & 6.25 \\
\hline 3 & 16 \\
\hline 4 & 40 \\
\hline 5 & 100 \\
\hline 6 & 250 \\
\hline
\end{tabular}

This suggests a question - what does it mean to have an apparent magnitude of zero? It's similar to asking if $0^{\circ} \mathrm{C}$ is the coldest temperature. In fact, a star of apparent magnitude zero is quite bright. Also, as $0^{\circ} \mathrm{C}$ was chosen to anchor the Celsius scale (at the freezing point of water), the star Vega was originally chosen to anchor the magnitude scale as the representative zero magnitude star. $^{1}$

A good way to teach this backwards notion-that smaller numbers count for more than larger numbers, and negative numbers even more so-is by using an inverted number line (see Fig. 1). This way, one can simply count the spaces between -2 and +3 to see they are five magnitudes apart. Another analogy that can help is to use sports such as golf, which uses lower scores to represent better results, although of course one can't have a negative score in golf (analogies do have their limits).

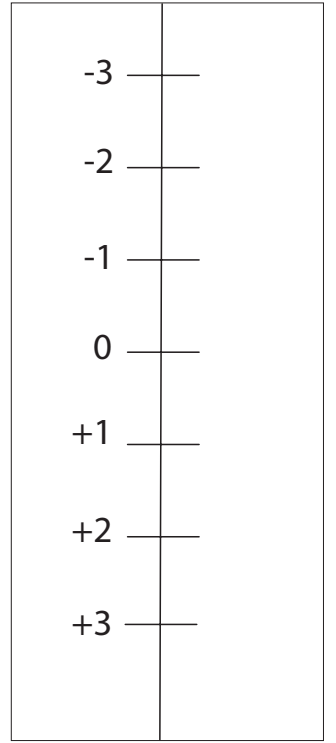

Fig. 1. Number line with negative numbers at the top rather than the bottom.
You may have noticed that I and other astronomers often use the word apparent in front of terms such as brightness or magnitude. This is not us being picky or pedantic. Apparent means what it says-"apparent brightness" is how bright a star appears to be to us.

Why does this matter? Because received light intensity (a.k.a. "flux" for astronomers) depends upon distance via the inverse square law:

$$
F=\frac{L}{4 \pi d^{2}},
$$

where $F$ stands for flux, $L$ for luminosity, and $d$ for distance. 
Astronomers use the term luminosity to represent the amount of light power emitted by a star (and in fact luminosity is often expressed in units of watts ${ }^{2}$ ). By contrast, flux is the amount of light power per unit area. In this vein, apparent magnitude corresponds to what we perceive, which is flux; astronomers use absolute magnitude to correspond to what the star actually emits, which is luminosity. ${ }^{3}$

Astronomers link these two versions of magnitudes via a definition: let absolute magnitude be the apparent magnitude of a star exactly 10 parsecs away. Absolute magnitude can be estimated by knowing other properties of the star (such as "spectral type" and "luminosity class"), and so, with both apparent and absolute magnitudes available, we can calculate the distance to the star in units of parsecs:

$$
d=10^{(m-M+5) / 5},
$$

where $m$ is apparent magnitude and $M$ is absolute magnitude.

How you use all this information to teach your students depends in large part on your audience. If your students are comfortable with base-10 logarithms and exponents, then simply give the students some sample numbers. Suppose a star has an apparent magnitude of +4 and an absolute magnitude of +1 . Then

$$
d=10^{(m-M+5) / 5}=10^{\frac{4-1+5}{5}}=10^{\frac{8}{5}}=10^{1.6} \approx 40 \mathrm{pc} .
$$

On the other hand, many of my astronomy students are math phobic. Here's a way to break this down, step-by-step, without the explicit use of logarithms:

(1) Is the star closer to us than $10 \mathrm{pc}$, farther from us than $10 \mathrm{pc}$, or exactly $10 \mathrm{pc}$ away?

Answer: Because the star looks dimmer $(+4)$ than it really is $(+1)$, it is farther away than $10 \mathrm{pc}$.
(2) What is the difference between the apparent and absolute magnitudes?

Answer: $4-1=3$.

(3) What is this difference in magnitude equivalent to regarding a ratio of brightness?

Answer: A difference of three magnitudes is equivalent to 16 times in brightness (see Table I).

(4) The brightness of a star decreases as the square of the distance. Therefore, to address distance, what is the square root of the ratio of brightness?

Answer: The square root of 16 is 4 .

(5) Finally, multiply this result (since the star is farther away than $10 \mathrm{pc}$ ) by $10 \mathrm{pc}$ to find the distance:

Answer: 4 times 10 pc equals 40 pc.

I wish I could say that the magnitude system is the only backwards, overly complicated bit of jargon offered by astronomy. The good news is that there is logic to astronomy, and while it may take some time to get used to, much of what we discuss can be digested (with some effort, true) by nonastronomers.

A sample activity involving magnitudes is available. Good luck and clear skies!

\section{References}

1. Arlo Landolt published observations of a large sample of stars in 1983 to serve as standard (calibration) stars. Updates to the Landolt system continue to this day.

2. Depending on who one talks with, you may find your astronomer using cgs ("centimeter-gram-seconds") units rather than SI units, so luminosity may be expressed in ergs per second. It's still all powers of ten, ultimately.

3. We are making an otherwise-unspoken simplification of absolute magnitude here by only considering brightnesses in visible light. The term astronomers use when considering brightness at all possible wavelengths of light is bolometric magnitude. 